\title{
异长叶烷基吡唑类衍生物的合成及其抗肿瘤、抗炎活性研究
}

\author{
苪 坚 $a$ 杨金来 ${ }^{a}$ 黄建峰 ${ }^{a}$ 王佳瑜 ${ }^{a}$ 徐 徐 $a, b, c$ \\ 徐海军 $a$ 王石发 $*, a, b, c$ \\ ( ${ }^{a}$ 南京林业大学化学工程学院 南京 210037) \\ ( ${ }^{b}$ 江苏省生物质能源与材料重点实验室 南京 210042) \\ (c) 广西林产化学与工程重点实验室 南宁 530008)
}

\begin{abstract}
摘要 以异长叶烷䣳为原料, 采用叔丁醇钾为催化剂, 与芳香醛进行羟醛缩合反应合成中间体 $\mathbf{2} \mathbf{a} \sim \mathbf{2} \mathbf{j}$, 再与水合肼进 行缩合、环化和脱氢芳构化反应，得到异长叶烷基吡唑类衍生物 $\mathbf{3 a} \sim 3 \mathbf{j}$, 终产物的得率为 $69.5 \% \sim 76.5 \%$. 采用 ${ }^{1} \mathrm{H} \mathrm{NMR}$, ${ }^{13} \mathrm{C} N M R$, IR, MS 和元素分析表征了化合物的结构, 通过 X 射线衍射分析测定了化合物 $\mathbf{3 i}$ 的晶体结构. 对合成所得化 合物进行了抗炎活性、抗肿瘤活性测定. 结果表明, 化合物 $\mathbf{3 h}$ 与 $\mathbf{3 i}$ 对人脐静脉内皮细胞(HUVECs)表现出显著的抗炎 活性，其 $\mathrm{IC}_{50}$ 值分别为 $(15.42 \pm 0.6)$ 和(4.74 \pm 0.3$) \mu \mathrm{mol} / \mathrm{L}$ ，化合物 $\mathbf{3 a}, \mathbf{3} \mathbf{c}, \mathbf{3 e}, \mathbf{3} \mathbf{j}$ 对人体肝癌细胞(HepG2)具有体外抗肿瘤 活性，其 $\mathrm{IC}_{50}$ 分别为 $(5.27 \pm 0.5),(6.71 \pm 0.4),(4.68 \pm 0.2)$ 和(4.57 \pm 0.5$) \mu \mathrm{mol} / \mathrm{L}$.
\end{abstract}

关键词 异长叶烷酮; 7-芳亚甲基异长叶烷酮; 吡唑衍生物; 抗炎活性; 抗肿瘤活性

\section{Synthesis, Antitumor and Anti-inflammatory Activities of Isolongifolanonyl Pyrazole Derivatives}

\author{
Rui, $\operatorname{Jian}^{a} \quad$ Yang, Jianlai $^{a} \quad$ Huang, Jianfeng $^{a} \quad$ Wang, Jiayu ${ }^{\mathrm{a}}$ \\ $\mathrm{Xu}, \mathrm{Xu}^{a, b, c} \quad \mathrm{Xu}$, Haijun $^{a} \quad$ Wang, Shifa ${ }^{*, a, b, c}$ \\ $\left({ }^{a}\right.$ College of Chemical Engineering, Nanjing Forestry University, Nanjing 210037) \\ ( ${ }^{b}$ Key Laboratory of Biomass Energy and Material of Jiangsu Province, Nanjing 210042) \\ ( ${ }^{c}$ Guangxi Key Laboratory of Chemistry and Engineering of Forest Products, Nanning 530008)
}

\begin{abstract}
A series of new isolongifolanonyl pyrazole derivatives $\mathbf{3 a} \sim \mathbf{3} \mathbf{j}$ were synthesized by aldol-condensation, cyclization and dehydroaromatization from isolongifolanone. The yields of products ranged from $69.5 \%$ to $76.5 \%$. The chemical structures of compounds obtained were elucidated by ${ }^{1} \mathrm{H}$ NMR, ${ }^{13} \mathrm{C}$ NMR, IR, MS spectra and elemental analysis. The strucure of compound 3i was determined by X-ray single crystal diffraction. Their inhibitory activities against human hepatoma cell (HepG2) and human umbilical vein endothelial cell (HUVECs) were evaluated. The results indicated that compounds $\mathbf{3 h}$ and $\mathbf{3 i}\left[\mathrm{IC} \mathrm{C}_{50}=\right.$ $(15.42 \pm 0.6),(4.74 \pm 0.3) \mu \mathrm{mol} / \mathrm{L}]$ showed obvious inhibitory activity against HUVECs. Compounds $\mathbf{3 a}, \mathbf{3 c}, \mathbf{3 e}$ and $\mathbf{3 j}\left[\mathrm{IC}_{50}=\right.$ $(5.27 \pm 0.5),(6.71 \pm 0.4),(4.68 \pm 0.2),(4.57 \pm 0.5)] \mu \mathrm{mol} / \mathrm{L}$ showed better antitumor activity against HepG2. Keywords isolongifolanone; 7-arylidene-isolongifolanone; pyrazole derivatives; anti-inflammatory activity; antitumor activity
\end{abstract}

吡唑类化合物作为杂环化合物的一个重要分支, 是 一类具有广谱生物活性的含氮杂环化合物 ${ }^{[1]}$, 具有较好 的生物活性及配位性能 ${ }^{[2,3]}$. 在医药方面, 该类化合物具 有消炎、抗高血糖、抗癌、抗病毒等 ${ }^{[49]}$ 作用; 在农业

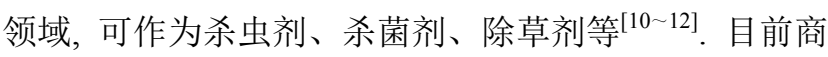
品化的吡唑类药物有消炎药塞来西布 ${ }^{[13]}$ 、杀菌剂唑菌胺 酯 ${ }^{[14]} 、$ 除草剂吡嘧磺隆 ${ }^{[15]}$ 等.

脂松节油是我国主要的森林资源化学品之一, 资源

\footnotetext{
*E-mail: wsfyyq@njfu.com.cn

Received March 14, 2016; revised April 11, 2016; published online May 6, 2016.

Project supported by the University Science Research Project of Jiangsu Province (No. 14KJ220001), the Natural National Science Foundation of China (No. 31470529), and the Open Funding of Jiangsu Key Laboratory of Biomass Energy and Materials (No. JSBEM2014010).

江苏省高校自然科学研究重大项目(No. 14KJ220001)、国家自然科学基金(No. 31470529)和江苏省生物质能源与材料重点实验室开放基金(No. JSBEM2014010)资助项目.
} 
丰富, 占世界脂松节油总量的 $80 \%$ 以上. 松节油的主要 成分之一长叶烯是一种三环倍半萜类化合物, 长叶烯基 衍生物具有良好的生物活性 ${ }^{[16 ~ 19]}$, 如抗肺结核、驱蚊、 驱虫等. 目前吡唑类衍生物成为抗肿瘤、抗炎新药物的 研究热点, 通过研究发现吡唑类衍生物可以作为蛋白酶 抑制剂, 从而对基因的表达进行控制，达到抗肿瘤、抗 炎的效果 ${ }^{[20,21]}$. Dinges 等 ${ }^{[2]}$ 发现 1,4-二氢狮并 $[1,2-c]$ 吡 唑可作为一类新的血管内皮生长因子受体 $(\mathrm{KDR})$ 激酶抑 制剂. 为了开发具有生物活性的长叶烯衍生物异长叶烷 酮, 本文采用活性结构拼接的方法, 将异长叶烷基、吡 唑环引入到同一个分子中, 设计合成了一系列新型异长 叶烷基吡唑类衍生物 $\mathbf{3 a} \sim \mathbf{3} \mathbf{j}$, 合成路线见 Scheme 1.

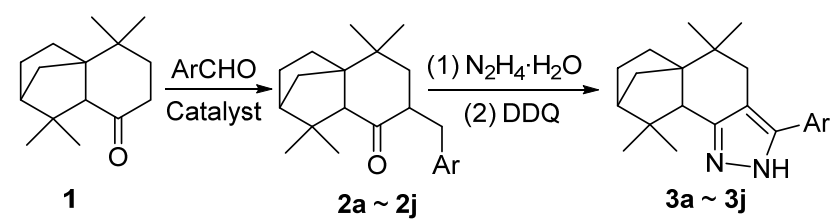

$\mathrm{Ar}=4-\mathrm{ClC}_{6} \mathrm{H}_{4}(\mathbf{a}), 4-\mathrm{BrC}_{6} \mathrm{H}_{4}(\mathbf{b}), 4-\mathrm{FC}_{6} \mathrm{H}_{4}(\mathbf{c}), 4-\mathrm{CH}_{3} \mathrm{C}_{6} \mathrm{H}_{4}($ d), 4- $\mathrm{CH}_{3} \mathrm{OC}_{6} \mathrm{H}_{4}(\mathbf{e}), \mathrm{C}_{6} \mathrm{H}_{5}(\mathbf{f}), 4-\mathrm{O}_{2} \mathrm{NC}_{6} \mathrm{H}_{4}(\mathbf{g}), 3-\mathrm{O}_{2} \mathrm{NC}_{6} \mathrm{H}_{4}$ (h), 4- $\left(\mathrm{CH}_{3}\right)_{2} \mathrm{NC}_{6} \mathrm{H}_{4}(\mathbf{i}), 2,4-\mathrm{Cl}_{2} \mathrm{C}_{6} \mathrm{H}_{3}(\mathrm{j})$

图式 1 目标化合物的合成

Scheme 1 Synthesis of target compounds

\section{1 结果与讨论}

\section{1 合成部分}

合成中间体 $\mathbf{2 a} \sim \mathbf{2} \mathbf{j}$ 的反应为经典的羟醛缩合反应, 芳香醛上取代基对羟醛缩合反应进行的难易程度影响 很大; 当苯环上有吸电子取代基(如 $\mathrm{F}, \mathrm{Cl}, \mathrm{Br}, \mathrm{NO}_{2}$ ) 时, 亲核加成反应较易进行, 反应时间短; 而当苯环上有供 电子取代基(如 $\mathrm{CH}_{3}, \mathrm{OCH}_{3}$ )时, 亲核加成反应相对较难 进行, 反应时间也增长.

在合成目标产物 $3 \mathbf{a} \sim 3 \mathbf{j}$ 过程中, 第一步完成后需 要除去剩余的水合肼, 防止降低脱氢芳构化反应效率. 第二步相对于 $\mathrm{KMnO}_{4} 、 \mathrm{H}_{2} \mathrm{O}_{2}$ 等氧化剂而言, 以 2,3-二 氯-5,6-二氰基苯醌(DDQ)作氧化剂, 转化率更高, 副反 应少.

\section{2 结构表征}

分析化合物的 ${ }^{1} \mathrm{H}$ NMR 数据发现, 中间体 $\mathbf{2 a} \sim \mathbf{2} \mathbf{j}$ 在 $\delta 7.32 \sim 7.53$ 之间的多重峰是芳亚甲基上氢的吸收信 号, 氢原子个数与预期一致. 中间体 $\mathbf{2 a} \sim \mathbf{2} \mathbf{j}$ 经缩合、环 化和脱氢芳构化反应后, 由 $\alpha, \beta$-不饱和酮转化成具有芳 香性结构的吡唑环, 此时吡唑环上 $\mathrm{NH}$ 的质子信号在 $\delta$ 12.40 附近, 而且是一个宽峰. 在 ${ }^{13} \mathrm{C} N \mathrm{~N} R$ 中, 中间体 $\mathbf{2 a} \sim \mathbf{2 j}$ 上羰基碳的化学位移出现在 $\delta 202.00$ 左右, 而目 标产物 $\mathbf{3 a} \sim 3 \mathbf{j}$ 结构中无羰基碳, 故在此位置无吸收信
号. 目标化合物 $\mathbf{3 a} \sim \mathbf{3} \mathbf{j}$ 的红外光谱中, 所有特征基团均 有明显的吸收, 在 $3400 \sim 3200 \mathrm{~cm}^{-1}$ 附近区域出现 $\mathrm{N}$ $\mathrm{H}$ 键的伸缩振动吸收峰; 3035 3180 $\mathrm{cm}^{-1}$ 之间出现芳基 $\mathrm{C}-\mathrm{H}$ 伸缩振动的特征吸收峰; $2836 \sim 2971 \mathrm{~cm}^{-1}$ 之间为 甲基及亚甲基 $\mathrm{C}-\mathrm{H}$ 键的伸缩振动吸收峰; 在 1439 $1598 \mathrm{~cm}^{-1}$ 区域有苯环骨架的伸缩振动吸收峰; 在 815 $855 \mathrm{c} \mathrm{m}^{-1}$ 区域的吸收峰为对位二取代苯环上 $\mathrm{C}-\mathrm{H}$ 的面外弯曲振动; $694,768 \mathrm{~cm}^{-1}$ 为单取代苯环上 $\mathrm{C}-\mathrm{H}$ 的面外弯曲振动; $723,803 \mathrm{~cm}^{-1}$ 为间位二取代苯 环上 $\mathrm{C}-\mathrm{H}$ 的面外弯曲振动; $809,867 \mathrm{~cm}^{-1}$ 为 $1,2,4$ 位三 取代苯环上 $\mathrm{C}-\mathrm{H}$ 的面外弯曲振动. 质谱数据可以看出, 目标分子均有正常的分子离子峰，而且元素分析数据的 理论值与计算值也符合得很好，其相对误差基本在 $0.4 \%$ 范围内. 据文献报道 ${ }^{[23]}$, 当吡唑环上的 1 -位无取代 基时，该类化合物之间就会存在互变异构现象; 晶体结 构分析表明，化合物 3i 晶体为正交晶系，空间群 $P 21212$ (图 1).

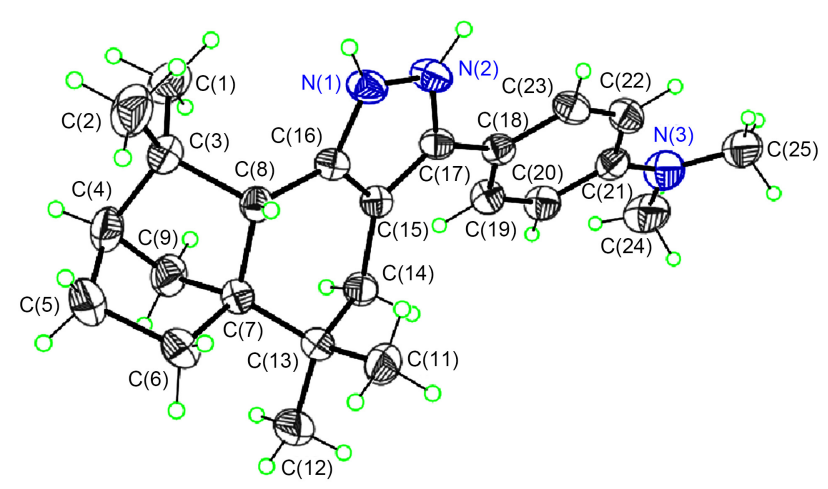

图 1 化合物 $3 \mathrm{i}$ 的分子结构图

Figure 1 Molecular structure of compound 3i

\section{3 化合物的抗肿瘤、抗炎活性}

对 10 个异长叶烷基吡唑类化合物分别进行人脐静 脉内皮细胞(HUVECs)的抗炎活性测试与人体肝癌细胞 (HepG2)的抗肿瘤活性测试, 其活性结果见表 1.

由表 1 可知，在化合物 $\mathbf{3 a} \sim \mathbf{3} \mathbf{j}$ 对 HUVECs 的抗炎 活性测试中, 化合物 $\mathbf{3 h}$ 与 $\mathbf{3 j}$ 对 HUVECs 细胞具有较好 的抗炎活性, 其 $\mathrm{IC}_{50}$ 值分别为 $(15.42 \pm 0.6)$ 和 $(4.74 \pm 0.3)$ $\mu \mathrm{mol} \cdot \mathrm{L}^{-1}$, 尤其是化合物 $\mathbf{3 i}$, 其抗炎活性值与对照药阿 司匹林相接近, 可以作为一种潜在的抗炎药物进行深入 研究. 通过抗炎活性研究发现, 含氮类吡唑化合物对 HUVECs 具有较好的抗炎活性, 其余化合物对其抑制效 果较差. 在对 HUVECs 的抗肿瘤活性测试中，化合物 3a, 3c, 3e, 3j 对 HepG2 具有较好的体外抗肿瘤活性 $\left[\mathrm{IC}_{50}=(5.27 \pm 0.5),(6.71 \pm 0.4),(4.68 \pm 0.2),(4.57 \pm 0.5)\right.$ $\left.\mu \mathrm{mol} \cdot \mathrm{L}^{-1}\right]$. 化合物 $\mathbf{3 a}, \mathbf{3 b}, \mathbf{3} \mathbf{c}, \mathbf{3} \mathbf{j}$ 均含有卤素官能团, 且 对 HepG2 均有抑制作用. 与化合物 $\mathbf{3 f}$ 相比，说明卤素官 
表 1 化合物 3a $\sim \mathbf{3 j}$ 对 HUVECs 的抗炎活性与 HepG2 的抗肿 瘤活性 $^{a}$

Table1 Antitumor and anti-inflammatory activities of compounds $\mathbf{3 a} \sim \mathbf{3} \mathbf{j}$ against HepG2 and HUVECs

\begin{tabular}{clll}
\hline \multirow{2}{*}{ 样品 } & \multicolumn{1}{c}{$\mathrm{R}$} & \multicolumn{2}{c}{$\mathrm{IC}_{50}{ }^{b} /\left(\mu \mathrm{mol} \cdot \mathrm{L}^{-1}\right)$} \\
\cline { 3 - 4 } & \multicolumn{1}{c}{$\mathrm{HepG} 2$} & $\mathrm{HUVECs}$ \\
\hline $\mathbf{3 a}$ & $4-\mathrm{ClC}_{6} \mathrm{H}_{4}$ & $5.27 \pm 0.5$ & $>120$ \\
$\mathbf{3 b}$ & $4-\mathrm{BrC}_{6} \mathrm{H}_{4}$ & $88.51 \pm 0.9$ & $>120$ \\
$\mathbf{3 c}$ & $4-\mathrm{FC}_{6} \mathrm{H}_{4}$ & $6.71 \pm 0.4$ & $>120$ \\
$\mathbf{3 d}$ & $3-\mathrm{CH}_{3} \mathrm{C}_{6} \mathrm{H}_{4}$ & $>120$ & $>120$ \\
$\mathbf{3 e}$ & $4-\mathrm{CH}_{3} \mathrm{OC}_{6} \mathrm{H}_{4}$ & $4.68 \pm 0.2$ & $>120$ \\
$\mathbf{3 f}$ & $\mathrm{C}_{6} \mathrm{H}_{5}$ & $>120$ & $>120$ \\
$\mathbf{3 g}$ & $4-\mathrm{O}_{2} \mathrm{NC}_{6} \mathrm{H}_{4}$ & $>120$ & $84.12 \pm 0.9$ \\
$\mathbf{3 h}$ & $3-\mathrm{O}_{2} \mathrm{NC}_{6} \mathrm{H}_{4}$ & $>120$ & $15.42 \pm 0.6$ \\
$\mathbf{3 i}$ & $4-\left(\mathrm{CH}_{3}\right)_{2} \mathrm{NC}_{6} \mathrm{H}_{4}$ & $>120$ & $4.74 \pm 0.3$ \\
$\mathbf{3 j}$ & $2,4-\mathrm{Cl}_{2} \mathrm{C}_{6} \mathrm{H}_{3}$ & $4.57 \pm 0.5$ & $>120$ \\
阿司匹林 & & $\mathrm{ND}$ & $5.1 \pm 0.2$ \\
依托泊苷 & & $3.5 \pm 0.2$ & $\mathrm{ND}$ \\
\hline
\end{tabular}

${ }^{a} \mathrm{ND}$ : 没有进行测试. $\mathrm{b}$ 细胞在含有目标化合物的培养基中连续培养 $24 \mathrm{~h}$.

${ }^{b}$ 所有数据均有 3 个平行样, 且 $\mathrm{IC}_{50}$ 值仅给出 $120 \mu \mathrm{mol} / \mathrm{L}$ 以下的.

能团或许是对 HepG2 起抑制作用的有效官能团. 化合 物 $3 \mathbf{a}, \mathbf{3 c}, \mathbf{3 e}, \mathbf{3} \mathbf{j}$ 可以作为潜在的抗肿瘤药物进行深入研 究, 为未来抗肿瘤药物的研发提供一定的实验依据.

\section{2 结论}

以异长叶烷酮为原料设计合成了 10 个新型异长叶 烷基吡唑类化合物 $\mathbf{3} \mathbf{a} \sim \mathbf{3} \mathbf{j}$, 采用 ${ }^{1} \mathrm{H} N \mathrm{NM},{ }^{13} \mathrm{C} \mathrm{NMR}, \mathrm{IR}$, MS, X-ray 和元素分析等手段表征所得化合物的结构, 并测试了化合物对 HUVECs 的抗炎活性与 HepG2 的抗 肿瘤活性测. 结果表明, 化合物 3h, 3i 对 HUVECs 炎性 反应具有显著的抑制作用, 且与阳性药阿司匹林的抗炎 活性相近. 化合物 3a, 3c, 3e, 3j 对 HepG2 具有较好的体 外抗肿瘤活性, 可以作为潜在的抗肿瘤药物进行深入研 究.

\section{3 实验部分}

\section{1 仪器与试剂}

美国 Thermo Electron Corporation Nicolet 380 FT-IR 红外光谱仪; 德国 Bruker AV 300/500/600 MHz 核磁共 振仪(TMS 为内标, $\mathrm{CDCl}_{3}-d_{6}$ 或 $\mathrm{DMSO}$ 为溶剂); 美国 Agilent 7890A 气相色谱仪; 美国 Agilent 5975C 质谱仪; 北京泰克 X-6 显微熔点测定仪(温度计未经校正); 德国 Elementar Vario EL cube 元素分析仪.

异长叶烷酮购自福建省武夷香化建阳香料香精公 司, 纯度 $80 \%$; 其余试剂均为市售分析纯, 未处理直接 使用.

细胞: 人体肝癌癌细胞(HepG2)和人脐静脉内皮细 胞(HUVECs)均由南京林业大学化学工程学院提供.

\section{2 实验方法}

\subsection{1中间体 $\mathbf{2} \mathbf{a} \sim \mathbf{2} \mathbf{j}$ 的制备}

将 $8 \mathrm{mmol}$ 异长叶烷酮、30 $\mathrm{mL}$ 叔丁醇、 $10 \mathrm{mmol}$ 苯甲醛和 $2 \sim 10 \mathrm{mmol}$ 叔丁醇钾依次加入配有搅拌器、 温度计和回流冷凝器的 $50 \mathrm{~mL}$ 三口烧瓶中, 搅拌均匀, 回流反应数小时, 至异长叶烷酮转化率达 $95 \%$ 以上 $(\mathrm{GC}$ 跟踪检测). 反应液用乙酸乙酯萃取, 经饱和食盐水洗 至中性, 无水 $\mathrm{Na}_{2} \mathrm{SO}_{4}$ 干燥、过滤、浓缩后得到粗产物, 再 经乙醇重结晶得中间体 $\mathbf{2} \mathbf{a} \sim \mathbf{2 j}$.

7-(4'-氯苯亚甲基)异长叶烷酮(2a): 得率 93.5\%. 黄 色针状晶体, m.p. 158.4 159.1 ${ }^{\circ} \mathrm{C} ;{ }^{1} \mathrm{H}$ NMR $\left(\mathrm{CDCl}_{3}\right.$, $600 \mathrm{MHz}) \delta: 0.85(\mathrm{~s}, 3 \mathrm{H}), 0.87(\mathrm{~s}, 3 \mathrm{H}), 1.06(\mathrm{~s}, 3 \mathrm{H})$, $1.08 \sim 1.13(\mathrm{~m}, 1 \mathrm{H}), 1.24(\mathrm{~s}, 3 \mathrm{H}), 1.31 \sim 1.32(\mathrm{~m}, 1 \mathrm{H})$, $1.49 \sim 1.55(\mathrm{~m}, 1 \mathrm{H}), 1.63 \sim 1.68(\mathrm{~m}, 1 \mathrm{H}), 1.78 \sim 1.81(\mathrm{~m}$, $2 \mathrm{H}), 1.83 \sim 1.87(\mathrm{~m}, 1 \mathrm{H}), 1.99(\mathrm{~d}, J=2.0 \mathrm{~Hz}, 1 \mathrm{H}), 2.55 \sim$ $2.58(\mathrm{~m}, 1 \mathrm{H}), 2.81 \sim 2.84(\mathrm{~m}, 1 \mathrm{H}), 7.37(\mathrm{~d}, J=8.6 \mathrm{~Hz}$, $2 \mathrm{H}), 7.41(\mathrm{~d}, J=8.6 \mathrm{~Hz}, 2 \mathrm{H}), 7.47(\mathrm{~s}, 1 \mathrm{H}) ;{ }^{13} \mathrm{C}$ NMR $\left(\mathrm{CDCl}_{3}, 150 \mathrm{MHz}\right) \delta: 24.13,24.67,25.53,25.72,28.36$, $30.28,31.73,37.62,41.65,44.83,48.08,55.54,62.95$, $128.65,131.79,134.36,134.48,135.19,135.83,202.61$; IR (KBr) v: 2950, 2895, 2869, 1670, 1595, 1487, 1464, 825 $\mathrm{cm}^{-1}$; MS (70 eV) m/z (\%): $342\left(\mathrm{M}^{+}, 90\right), 327$ (23), 299 (100). Anal. calcd for $\mathrm{C}_{22} \mathrm{H}_{27} \mathrm{ClO}$ : $\mathrm{C} 77.06, \mathrm{H} 7.94$; found C 77.12, H 7.97.

7-(4'-溴苯亚甲基)异长叶烷酮(2b): 得率 91.8\%. 黄 色针状晶体, m.p. $129.7 \sim 130.2{ }^{\circ} \mathrm{C} ;{ }^{1} \mathrm{H}$ NMR $\left(\mathrm{CDCl}_{3}\right.$, $600 \mathrm{MHz}) \delta: 0.85(\mathrm{~s}, 3 \mathrm{H}), 0.87$ (s, 3H), $1.08(\mathrm{~s}, 3 \mathrm{H})$, $1.09 \sim 1.13(\mathrm{~m}, 1 \mathrm{H}), 1.24(\mathrm{~s}, 3 \mathrm{H}), 1.31(\mathrm{~d}, J=10.3 \mathrm{~Hz}$, $1 \mathrm{H}), 1.49 \sim 1.55(\mathrm{~m}, 1 \mathrm{H}), 1.63 \sim 1.68(\mathrm{~m}, 1 \mathrm{H}), 1.78 \sim 1.80$ $(\mathrm{m}, 2 \mathrm{H}), 1.82 \sim 1.87(\mathrm{~m}, 1 \mathrm{H}), 1.99(\mathrm{~d}, J=1.8 \mathrm{~Hz}, 1 \mathrm{H})$, $2.54 \sim 2.57(\mathrm{~m}, 1 \mathrm{H}), 2.79 \sim 2.82(\mathrm{~m}, 1 \mathrm{H}), 7.33(\mathrm{~d}, J=8.4$ $\mathrm{Hz}, 2 \mathrm{H}), 7.44$ (s, 1H), 7.52 (d, $J=8.4 \mathrm{~Hz}, 2 \mathrm{H}) ;{ }^{13} \mathrm{C} \mathrm{NMR}$ $\left(\mathrm{CDCl}_{3}, 150 \mathrm{MHz}\right) \delta: 24.13,24.68,25.54,25.72,28.36$, $30.29,31.73,37.62,41.64,44.84,48.08,55.54,62.95$, $122.82,131.61,131.85,132.01,132.20,134.80,135.23$, 135.97, 202.56; IR (KBr) v: 2947, 2895, 2871, 1670, 1598, 1483, 1464, $816 \mathrm{~cm}^{-1}$; MS (70 eV) m/z (\%): $387\left(\mathrm{M}^{+}, 42\right)$, 372(5), 345(100). Anal. calcd for $\mathrm{C}_{22} \mathrm{H}_{27} \mathrm{BrO}$ : $\mathrm{C} 68.22, \mathrm{H}$ 7.03; found C 68.15, H 7.11.

7-(4'-氟苯亚甲基)异长叶烷酮(2c): 得率 92.4\%. 乳 白色针状晶体, m.p. 139.7 140.3 ${ }^{\circ} \mathrm{C} ;{ }^{1} \mathrm{H}$ NMR $\left(\mathrm{CDCl}_{3}\right.$, $500 \mathrm{MHz}) \delta: 0.85(\mathrm{~s}, 3 \mathrm{H}), 0.87(\mathrm{~s}, 3 \mathrm{H}), 1.07(\mathrm{~s}, 3 \mathrm{H})$, $1.10 \sim 1.13(\mathrm{~m}, 1 \mathrm{H}), 1.24(\mathrm{~s}, 3 \mathrm{H}), 1.31(\mathrm{~d}, J=10.0 \mathrm{~Hz}$, $1 \mathrm{H}), 1.48 \sim 1.55(\mathrm{~m}, 1 \mathrm{H}), 1.63 \sim 1.68(\mathrm{~m}, 1 \mathrm{H}), 1.78 \sim 1.81$ $(\mathrm{m}, 2 \mathrm{H}), 1.83 \sim 1.87(\mathrm{~m}, 1 \mathrm{H}), 1.99(\mathrm{~d}, J=1.9 \mathrm{~Hz}, 1 \mathrm{H})$, 
$2.56 \sim 2.59(\mathrm{~m}, 1 \mathrm{H}), 2.81 \sim 2.84(\mathrm{~m}, 1 \mathrm{H}), 7.08\left(\mathrm{t}, J_{\mathrm{H}-\mathrm{F}}=\right.$ $9.1 \mathrm{~Hz}, 2 \mathrm{H}), 7.47\left(\mathrm{dd}, J_{\mathrm{H}-\mathrm{F}}=8.7, J_{\mathrm{H}-\mathrm{H}}=6.1 \mathrm{~Hz}, 2 \mathrm{H}\right) ; 7.49$ (s, $1 \mathrm{H}) ;{ }^{13} \mathrm{C}$ NMR $\left(\mathrm{CDCl}_{3}, 125 \mathrm{MHz}\right) \delta: 24.05,24.62$, 25.46, 25.66, 28.26, 30.26, 31.65, 37.56, 41.56, 44.74, $48.08,55.52,62.93,115.42\left(\mathrm{~d}, J_{\mathrm{C}-\mathrm{F}}=10.6 \mathrm{~Hz}, 1 \mathrm{C}\right), 132.09$ $\left(\mathrm{d}, J_{\mathrm{C}-\mathrm{F}}=3.7 \mathrm{~Hz}, 1 \mathrm{C}\right), 132.37\left(\mathrm{~d}, J_{\mathrm{C}-\mathrm{F}}=8.6 \mathrm{~Hz}, 1 \mathrm{C}\right)$, $135.00\left(\mathrm{~d}, J_{\mathrm{C}-\mathrm{F}}=2.2 \mathrm{~Hz}, 1 \mathrm{C}\right), 135.29\left(\mathrm{~d}, J_{\mathrm{C}-\mathrm{F}}=3.3 \mathrm{~Hz}\right.$, $1 \mathrm{C}), 162.56\left(\mathrm{~d}, J_{\mathrm{C}-\mathrm{F}}=248.7 \mathrm{~Hz}, 1 \mathrm{C}\right), 202.39$; IR (KBr) $v$ : 2954, 2923, 2891, 2872, 1669, 1597, 1505, 1463, 833 $\mathrm{cm}^{-1}$; MS (70 eV) $m / z(\%): 326\left(\mathrm{M}^{+}, 84\right), 311(23), 283$ (100). Anal. calcd for $\mathrm{C}_{22} \mathrm{H}_{27} \mathrm{FO}$ : C 80.94, H 8.34; found C 81.05, H 8.21.

7-(4'-甲基苯亚甲基)异长叶烷酮(2d): 得率 92.3\%. 淡黄色棉状固体, m.p. $120.4 \sim 121.2{ }^{\circ} \mathrm{C} ;{ }^{1} \mathrm{H}$ NMR $\left(\mathrm{CDCl}_{3}, 600 \mathrm{MHz}\right) \delta: 0.85(\mathrm{~s}, 3 \mathrm{H}), 0.88(\mathrm{~s}, 3 \mathrm{H}), 1.06(\mathrm{~s}$, $3 \mathrm{H}), 1.09 \sim 1.13(\mathrm{~m}, 1 \mathrm{H}), 1.24(\mathrm{~s}, 3 \mathrm{H}), 1.30(\mathrm{~d}, J=9.9 \mathrm{~Hz}$, $1 \mathrm{H}), 1.48 \sim 1.54(\mathrm{~m}, 1 \mathrm{H}), 1.63 \sim 1.65(\mathrm{~m}, 1 \mathrm{H}), 1.77(\mathrm{~d}, J=$ $4.1 \mathrm{~Hz}, 1 \mathrm{H}), 1.80 \sim 1.87(\mathrm{~m}, 2 \mathrm{H}), 1.98(\mathrm{~d}, J=1.9 \mathrm{~Hz}, 1 \mathrm{H})$, $2.38\left(\mathrm{~s}, 3 \mathrm{H}, \mathrm{Ar}-\mathrm{CH}_{3}\right), 2.61 \sim 2.64(\mathrm{~m}, 1 \mathrm{H}), 2.83 \sim 2.87(\mathrm{~m}$, $1 \mathrm{H}), 7.21(\mathrm{~d}, J=8.0 \mathrm{~Hz}, 2 \mathrm{H}), 7.40(\mathrm{~d}, J=8.0 \mathrm{~Hz}, 2 \mathrm{H}), 7.50$ (s, $1 \mathrm{H}) ;{ }^{13} \mathrm{C}$ NMR $\left(\mathrm{CDCl}_{3}, 150 \mathrm{MHz}\right) \delta: 21.44,24.13$, $24.70,25.51,25.75,28.40,30.27,31.69,37.60,41.81$, 44.76, 48.11, 55.57, 62.96, 129.17, 130.74, 133.14, 134.58, 136.64, 138.86, 202.89; IR (KBr) v: 2953, 2895, 2872, $1669,1591,1508,1466,816 \mathrm{~cm}^{-1}$; MS (70 eV) $\mathrm{m} / \mathrm{z}(\%)$ : $322\left(\mathrm{M}^{+}, 59\right), 307$ (100), 279 (37). Anal. calcd for $\mathrm{C}_{23} \mathrm{H}_{30} \mathrm{O}$ : C 85.66, H 9.38; found C 85.79, H 9.41.

7-(4'- 甲氧基苯亚甲基) 异长叶烷酮 (2e): 得率 $86.8 \%$. 黄色块状晶体, m.p. $112.4 \sim 113.1{ }^{\circ} \mathrm{C} ;{ }^{1} \mathrm{H}$ NMR $\left(\mathrm{CDCl}_{3}, 600 \mathrm{MHz}\right) \delta: 0.86(\mathrm{~s}, 3 \mathrm{H}), 0.87(\mathrm{~s}, 3 \mathrm{H}), 1.07$ (s, $3 \mathrm{H}), 1.09 \sim 1.13(\mathrm{~m}, 1 \mathrm{H}), 1.24(\mathrm{~s}, 3 \mathrm{H}), 1.30(\mathrm{~d}, J=9.9 \mathrm{~Hz}$, $1 \mathrm{H}), 1.48 \sim 1.54(\mathrm{~m}, 1 \mathrm{H}), 1.63 \sim 1.68(\mathrm{~m}, 1 \mathrm{H}), 1.77(\mathrm{~d}, J=$ $4.0 \mathrm{~Hz}, 1 \mathrm{H}), 1.80 \sim 1.86(\mathrm{~m}, 2 \mathrm{H}), 1.98(\mathrm{~d}, J=1.5 \mathrm{~Hz}, 1 \mathrm{H})$, $2.60 \sim 2.63(\mathrm{~m}, 1 \mathrm{H}), 2.83 \sim 2.86(\mathrm{~m}, 1 \mathrm{H}), 3.85(\mathrm{~s}, 3 \mathrm{H}$, Ar- $\left.-\mathrm{OCH}_{3}\right), 6.93(\mathrm{~d}, J=8.7 \mathrm{~Hz}, 2 \mathrm{H}), 7.47(\mathrm{~s}, 1 \mathrm{H}), 7.48 \sim$ $7.49(\mathrm{~m}, 2 \mathrm{H}) ;{ }^{13} \mathrm{C}$ NMR $\left(\mathrm{CDCl}_{3}, 150 \mathrm{MHz}\right) \delta: 24.17$, 24.71, 25.49, 25.78, 28.41, 30.24, 31.66, 37.58, 41.94, 44.71, 48.10, 55.32, 55.53, 62.91, 113.91, 128.64, 132.53, 133.29, 136.36, 159.93, 202.78; IR (KBr) v: 2952, 2895, 2872, 1662, 1586, 1510, 1463, 1252, 1050, $829 \mathrm{~cm}^{-1}$; MS (70 eV) $\mathrm{m} / z$ (\%): $338\left(\mathrm{M}^{+}, 100\right), 323$ (21), 295 (31). Anal. calcd for $\mathrm{C}_{23} \mathrm{H}_{30} \mathrm{O}_{2}$ : C 81.61, H 8.93; found C 81.82, H 9.07 .

7-苯亚甲基异长叶烷酮(2f): 得率 $88.1 \%$. 淡黄色块 状固体, m.p. 94.1 95.0 ${ }^{\circ} \mathrm{C} ;{ }^{1} \mathrm{H}$ NMR $\left(\mathrm{CDCl}_{3}, 600 \mathrm{MHz}\right)$ $\delta: 0.86$ (s, 3H), 0.88 (s, 3H), 1.06(s, 3H), $1.09 \sim 1.13(\mathrm{~m}$, $1 \mathrm{H}), 1.24(\mathrm{~s}, 3 \mathrm{H}), 1.31(\mathrm{~d}, J=9.9 \mathrm{~Hz}, 1 \mathrm{H}), 1.48 \sim 1.55(\mathrm{~m}$, $1 \mathrm{H}), 1.63 \sim 1.67(\mathrm{~m}, 1 \mathrm{H}), 1.78(\mathrm{~d}, J=4.2 \mathrm{~Hz}, 1 \mathrm{H}), 1.80 \sim$ $1.87(\mathrm{~m}, 2 \mathrm{H}), 1.99(\mathrm{~d}, J=1.9 \mathrm{~Hz}, 1 \mathrm{H}), 2.61 \sim 2.64(\mathrm{~m}$, $1 \mathrm{H}), 2.85 \sim 2.88(\mathrm{~m}, 1 \mathrm{H}), 7.32 \sim 7.35(\mathrm{~m}, 1 \mathrm{H}), 7.39 \sim 7.42$ (m, 2H), 7.49 (s, $J=7.5 \mathrm{~Hz}, 2 \mathrm{H}), 7.53(\mathrm{~s}, 1 \mathrm{H}) ;{ }^{13} \mathrm{C} \mathrm{NMR}$ $\left(\mathrm{CDCl}_{3}, 150 \mathrm{MHz}\right) \delta: 24.12,24.69,25.52,25.73,28.38$, $30.29,31.71,37.62,41.68,44.80,48.10,55.59,62.98$, $128.40,128.59,130.63,135.39,135.95,136.58,202.82$; IR (KBr) $v: 2955,2895,2873,1668,1591,1463,1444,755$, $692 \mathrm{~cm}^{-1}$; MS (70 eV) $\mathrm{m} / z(\%): 308\left(\mathrm{M}^{+}, 100\right), 293$ (17), 265 (64). Anal. calcd for $\mathrm{C}_{22} \mathrm{H}_{28} \mathrm{O}: \mathrm{C} 85.66, \mathrm{H} 9.15$; found C 85.39, H 9.21.

7-(4'-硝基苯亚甲基)异长叶烷酮(2g)：得率 85.4\%. 黄色棉状晶体, m.p. 177.4 178.0 ${ }^{\circ} \mathrm{C} ;{ }^{1} \mathrm{H}$ NMR $\left(\mathrm{CDCl}_{3}\right.$, $600 \mathrm{MHz}) \delta: 0.86(\mathrm{~s}, 3 \mathrm{H}), 0.89$ (s, 3H), 1.07(s, 3H), 1.10 $1.15(\mathrm{~m}, 1 \mathrm{H}), 1.25(\mathrm{~s}, 3 \mathrm{H}), 1.33 \sim 1.35(\mathrm{~m}, 1 \mathrm{H}), 1.51 \sim$ $1.57(\mathrm{~m}, 1 \mathrm{H}), 1.65 \sim 1.70(\mathrm{~m}, 1 \mathrm{H}), 1.80 \sim 1.81(\mathrm{~m}, 2 \mathrm{H})$, $1.84 \sim 1.89(\mathrm{~m}, 1 \mathrm{H}), 2.02(\mathrm{~d}, J=1.98 \mathrm{~Hz}, 1 \mathrm{H}), 2.55 \sim 2.58$ (m, $1 \mathrm{H}), 2.85 \sim 2.89(\mathrm{~m}, 1 \mathrm{H}), 7.53(\mathrm{~s}, 1 \mathrm{H}), 7.60(\mathrm{~d}, J=8.7$ $\mathrm{Hz}, 2 \mathrm{H}), 8.25$ (d, $J=8.8 \mathrm{~Hz}, 2 \mathrm{H}) ;{ }^{13} \mathrm{C} \mathrm{NMR}\left(\mathrm{CDCl}_{3}, 150\right.$ MHz) $\delta: 24.16,24.64,25.58,25.68,28.31,30.33,31.82$, $37.67,41.60,44.99,48.08,55.55,63.00,123.61,130.93$, 133.76, 138.65, 142.46, 147.12, 202.24; IR (KBr) v: 2965, 2923, 2875, 1672, 1601, 1518, 1466, 1345, $851 \mathrm{~cm}^{-1}$; MS (70 eV) $\mathrm{m} / z$ (\%): $353\left(\mathrm{M}^{+}, 49\right), 338$ (17), 310 (100). Anal. calcd for $\mathrm{C}_{22} \mathrm{H}_{27} \mathrm{NO}_{3}$ : C 74.76, $\mathrm{H}$ 7.70, N 3.96; found C 74.72, H 7.91, N 3.91.

7-(3'-硝基苯亚甲基)异长叶烷酮(2h): 得率 83.9\%. 褐色块状晶体, m.p. 87.3 87.6 ${ }^{\circ} \mathrm{C} ;{ }^{1} \mathrm{H}$ NMR $\left(\mathrm{CDCl}_{3}\right.$, $600 \mathrm{MHz}) \delta: 0.86(\mathrm{~s}, 3 \mathrm{H}), 0.89(\mathrm{~s}, 3 \mathrm{H}), 1.08(\mathrm{~s}, 3 \mathrm{H})$, $1.10 \sim 1.15(\mathrm{~m}, 1 \mathrm{H}), 1.26(\mathrm{~s}, 3 \mathrm{H}), 1.34 \sim 1.36(\mathrm{~m}, 1 \mathrm{H})$, $1.51 \sim 1.57(\mathrm{~m}, 1 \mathrm{H}), 1.65 \sim 1.70(\mathrm{~m}, 1 \mathrm{H}), 1.81 \sim 1.83(\mathrm{~m}$, $2 \mathrm{H}), 1.84 \sim 1.88(\mathrm{~m}, 1 \mathrm{H}), 2.02(\mathrm{~d}, J=1.7 \mathrm{~Hz}, 1 \mathrm{H}), 2.55 \sim$ $2.58(\mathrm{~m}, 1 \mathrm{H}), 2.88 \sim 2.92(\mathrm{~m}, 1 \mathrm{H}), 7.53(\mathrm{~s}, 1 \mathrm{H}), 7.57 \sim$ $7.60(\mathrm{~m}, 1 \mathrm{H}), 7.75(\mathrm{~d}, J=7.7 \mathrm{~Hz}, 1 \mathrm{H}), 8.19(\mathrm{~d}, J=8.2 \mathrm{~Hz}$, $1 \mathrm{H}), 8.32(\mathrm{~s}, 1 \mathrm{H}) ;{ }^{13} \mathrm{C} \mathrm{NMR}\left(\mathrm{CDCl}_{3}, 150 \mathrm{MHz}\right) \delta: 24.16$, $24.65,25.55,25.67,28.32,30.32,31.80,37.68,41.41$, 44.96, 48.07, 55.55, 62.97, 123.02, 124.54, 129.42, 133.63, 136.27, 137.54, 137.86, 148.24, 202.23; IR (KBr) v: 2962, 2895, 2871, 1669, 1600, 1528, 1464, 1348, 825, $720 \mathrm{~cm}^{-1}$; MS (70 eV) m/z (\%): $353\left(\mathrm{M}^{+}, 47\right), 338$ (17), 310 (100). Anal. calcd for $\mathrm{C}_{22} \mathrm{H}_{27} \mathrm{NO}_{3}$ : C 74.76, H 7.70, N 3.96; found C 74.86, H 7.58, N 4.01.

7-(4'-二甲氨基苯亚甲基)异长叶烷酮 (2i): 得率 
90.4\%. 橙色粉状晶体, m.p. $119.5 \sim 120.2{ }^{\circ} \mathrm{C}$; ${ }^{1} \mathrm{H}$ NMR $\left(\mathrm{CDCl}_{3}, 600 \mathrm{MHz}\right) \delta: 0.86(\mathrm{~s}, 3 \mathrm{H}), 0.87(\mathrm{~s}, 3 \mathrm{H}), 1.07(\mathrm{~s}$, $3 \mathrm{H}), 1.09 \sim 1.12(\mathrm{~m}, 1 \mathrm{H}), 1.24(\mathrm{~s}, 3 \mathrm{H}), 1.28 \sim 1.30(\mathrm{~m}$, $1 \mathrm{H}), 1.47 \sim 1.53(\mathrm{~m}, 1 \mathrm{H}), 1.62 \sim 1.67(\mathrm{~m}, 1 \mathrm{H}), 1.75 \sim 1.76$ $(\mathrm{m}, 1 \mathrm{H}), 1.79 \sim 1.86(\mathrm{~m}, 2 \mathrm{H}), 1.96(\mathrm{~d}, J=1.9 \mathrm{~Hz}, 1 \mathrm{H})$, $2.63(\mathrm{~d}, J=16.7 \mathrm{~Hz}, 1 \mathrm{H}), 2.83 \sim 2.86(\mathrm{~m}, 1 \mathrm{H}), 3.03(\mathrm{~s}, 6 \mathrm{H}$, $\left.\mathrm{Ar}-\mathrm{N}\left(\mathrm{CH}_{3}\right)_{2}\right), 6.74(\mathrm{~s}, 2 \mathrm{H}), 7.46 \sim 7.49(\mathrm{~m}, 3 \mathrm{H}) ;{ }^{13} \mathrm{C} \mathrm{NMR}$ $\left(\mathrm{CDCl}_{3}, 150 \mathrm{MHz}\right) \delta: 24.22,24.75,25.49,25.84,28.47$, $30.23,31.63,37.55,40.17,42.29,44.62,48.13,55.52$, $62.86,111.65,123.92,130.94,132.79,137.33,150.42$, 202.63; IR (KBr) v: 2960, 2923, 2891, 2872, 1656, 1606, 1565, 1522, 1466, 1440, $822 \mathrm{~cm}^{-1}$; MS (70 eV) $\mathrm{m} / z(\%)$ : $351\left(\mathrm{M}^{+}, 100\right), 336$ (5), 282 (10). Anal. calcd for $\mathrm{C}_{24} \mathrm{H}_{33} \mathrm{NO}$ : C 82.00, H 9.46, N 3.98; found $\mathrm{C} 80.97, \mathrm{H}$ $9.52, \mathrm{~N} 3.82$.

7-(2',4'-二氯苯亚甲基)异长叶烷酮 $(\mathbf{2 j})$ : 得率 $86.7 \%$. 淡黄色块状晶体, m.p. $127.7 \sim 128.1{ }^{\circ} \mathrm{C} ;{ }^{1} \mathrm{H} \mathrm{NMR}$ $\left(\mathrm{CDCl}_{3}, 600 \mathrm{MHz}\right) \delta: 0.77(\mathrm{~s}, 3 \mathrm{H}), 0.85(\mathrm{~s}, 3 \mathrm{H}), 0.96(\mathrm{~s}$, $3 \mathrm{H}), 1.08 \sim 1.11(\mathrm{~m}, 1 \mathrm{H}), 1.18(\mathrm{~s}, 3 \mathrm{H}), 1.25(\mathrm{~d}, J=9.9 \mathrm{~Hz}$, $1 \mathrm{H}), 1.45 \sim 1.51(\mathrm{~m}, 1 \mathrm{H}), 1.57 \sim 1.63(\mathrm{~m}, 1 \mathrm{H}), 1.75 \sim 1.79$ (m, 2H), 1.82 (d, J=10.0 Hz, 1H), 1.92 (s, 1H), 2.32 (d, $J=16.7 \mathrm{~Hz}, 1 \mathrm{H}), 2.75 \sim 2.79(\mathrm{~m}, 1 \mathrm{H}), 7.42(\mathrm{~s}, 1 \mathrm{H}), 7.47 \sim$ $7.49(\mathrm{~m}, 1 \mathrm{H}), 7.53$ (d, $J=8.4 \mathrm{~Hz}, 1 \mathrm{H}), 7.68(\mathrm{~s}, 1 \mathrm{H}) ;{ }^{13} \mathrm{C}$ $\mathrm{NMR}\left(\mathrm{CDCl}_{3}, 150 \mathrm{MHz}\right) \delta: 24.65,25.60,25.66,28.34$, $30.45,31.92,37.69,40.78,44.87,48.16,55.70,63.12$, $126.71,129.61,130.99,132.45,133.03,134.52,135.74$, 137.72, 202.26; IR (KBr) v: 2960, 2895, 2871, 1672, 1600, 1466, 867, $818 \mathrm{~cm}^{-1}$; MS (70 eV) $\mathrm{m} / z(\%): 377\left(\mathrm{M}^{+}, 2\right)$, 362 (1), 341 (100). Anal. calcd for $\mathrm{C}_{22} \mathrm{H}_{26} \mathrm{Cl}_{2} \mathrm{O}: \mathrm{C} 70.03, \mathrm{H}$ 6.95; found C 70.17, H 7.14.

\subsection{2 目标化合物 $3 \mathbf{a} \sim \mathbf{3} \mathbf{j}$ 的制备}

在单口烧瓶中, 依次加入 $12 \mathrm{mmol}$ 中间体 $\mathbf{2 a} \sim \mathbf{2} \mathbf{j}$ 、 $72 \mathrm{mmol}$ 水合肼 $(80 \%) 、 80 \mathrm{~mL}$ 无水乙醇, 在电磁搅拌下 加热升温, 回流反应 $10 \mathrm{~h}$. 随后将反应液冷却至室温, 加入 $20 \mathrm{~mL}$ 乙腈, 油浴旋蒸除去反应液中的溶剂和过量 的水合肼; 然后再依次加入 $70 \mathrm{~mL}$ 干燥后的 1,4-二氧六 环、13.2 mmol 2,3-二氯-5,6-二氧对苯醌(DDQ), 回流反 应 6 8 h; 然后冷却至室温, 向反应液中加入 $30 \mathrm{~mL}$ $20 \% \mathrm{KOH}$ 水溶液、 $80 \mathrm{~mL}$ 二氯甲烷, 室温搅拌 $0.5 \mathrm{~h}$, 再 用二氯甲烷萃取, 合并有机相用去离子水洗至中性; 经 无水硫酸钠干燥、过滤、浓缩后得到油状初产物, 采用 硅胶色谱柱纯化 $[V$ (石油醚) : $V$ (乙酸乙酯 $)=3: 1]$, 乙 醇重结晶, 得到目标化合物 $\mathbf{3 a} \sim \mathbf{3 j}$.

3-(4'-氯苯基)-5,5,9,9-四甲基- $2,4,5,6,7,8,9,9 \mathrm{a}$-八 氢-5a,8-亚甲基桥苯并吲唑(3a): 得率 $70.3 \%$. 粉色棉状
固体, m.p. $239.8 \sim 240.2{ }^{\circ} \mathrm{C} ;{ }^{1} \mathrm{H}$ NMR (DMSO- $d_{6}, 500$ $\mathrm{MHz}) \delta: 0.66(\mathrm{~s}, 3 \mathrm{H}), 0.75(\mathrm{~s}, 3 \mathrm{H}), 1.05(\mathrm{~s}, 3 \mathrm{H}), 1.13 \sim$ $1.15(\mathrm{~m}, 1 \mathrm{H}), 1.20(\mathrm{~d}, J=9.7 \mathrm{~Hz}, 1 \mathrm{H}), 1.26(\mathrm{~s}, 3 \mathrm{H}), 1.44 \sim$ $1.48(\mathrm{~m}, 1 \mathrm{H}), 1.57(\mathrm{~d}, J=9.6 \mathrm{~Hz}, 1 \mathrm{H}), 1.67 \sim 1.71(\mathrm{~m}$, $2 \mathrm{H}), 1.80 \sim 1.84(\mathrm{~m}, 1 \mathrm{H}), 2.23(\mathrm{~s}, 1 \mathrm{H}), 2.31(\mathrm{~d}, J=15.2$ $\mathrm{Hz}, 1 \mathrm{H}), 2.63(\mathrm{~d}, J=15.1 \mathrm{~Hz}, 1 \mathrm{H}), 7.45$ (d, $J=7.6 \mathrm{~Hz}$, 2H), 7.66 (d, $J=6.5 \mathrm{~Hz}, 2 \mathrm{H}), 12.47$ (brs, $1 \mathrm{H}, \mathrm{NH}) ;{ }^{13} \mathrm{C}$ $\mathrm{NMR}\left(\mathrm{CDCl}_{3}, 75 \mathrm{MHz}\right) \delta$ : 23.46, 25.31, 25.99, 27.20, 28.29, 29.53, 34.20, 35.93, 36.71, 42.09, 48.31, 50.64, $56.46,112.04,128.13,128.59,132.05,132.99$; IR (KBr) $v$ : 3216, 2971, 2873, 1491, 1455, $828 \mathrm{~cm}^{-1}$; MS (70 eV) $\mathrm{m} / z$ (\%): $354\left(\mathrm{M}^{+}, 97\right), 339$ (15), 271 (100). Anal. calcd for $\mathrm{C}_{22} \mathrm{H}_{27} \mathrm{ClN}_{2}$ : C 74.45, $\mathrm{H} 7.67, \mathrm{~N} 7.89$; found $\mathrm{C} 74.57, \mathrm{H}$ $7.74, \mathrm{~N} 8.00$.

3-(4'-溴苯基)-5,5,9,9-四甲基- $2,4,5,6,7,8,9,9 \mathrm{a}$-八氢 -5a,8-亚甲基桥苯并吲唑(3b)：得率 $71.2 \%$. 白色棉状固 体, m.p. 249.7 250.3 ${ }^{\circ} \mathrm{C}$; ${ }^{1} \mathrm{H}$ NMR (DMSO- $d_{6}, 500$ $\mathrm{MHz}) \delta$ : $0.65(\mathrm{~s}, 3 \mathrm{H}), 0.75(\mathrm{~s}, 3 \mathrm{H}), 1.05(\mathrm{~s}, 3 \mathrm{H}), 1.13 \sim$ $1.15(\mathrm{~m}, 1 \mathrm{H}), 1.20(\mathrm{~d}, J=9.7 \mathrm{~Hz}, 1 \mathrm{H}), 1.25(\mathrm{~s}, 3 \mathrm{H}), 1.44 \sim$ $1.48(\mathrm{~m}, 1 \mathrm{H}), 1.57(\mathrm{~d}, J=9.6 \mathrm{~Hz}, 1 \mathrm{H}), 1.67 \sim 1.70(\mathrm{~m}$, $2 \mathrm{H}), 1.80 \sim 1.84(\mathrm{~m}, 1 \mathrm{H}), 2.23(\mathrm{~s}, 1 \mathrm{H}), 2.31(\mathrm{~d}, J=15.2$ $\mathrm{Hz}, 1 \mathrm{H}), 2.62$ (d, $J=15.2 \mathrm{~Hz}, 1 \mathrm{H}), 7.59$ (s, 4H), 12.48 (brs, $1 \mathrm{H}, \mathrm{NH}) ;{ }^{13} \mathrm{C} \mathrm{NMR}\left(\mathrm{CDCl}_{3}, 75 \mathrm{MHz}\right) \delta: 23.46,25.31$, $25.99,27.18,28.26,29.52,34.20,35.92,36.71,42.08$, $48.30,50.61,56.45,112.06,121.15,128.45,131.53$, 132.48; IR (KBr) v: 3200, 2964, 2874, 1485, 1462, 827 $\mathrm{cm}^{-1}$; MS (70 eV) m/z (\%): $398\left(\mathrm{M}^{+}, 100\right), 383$ (13), 317(93). Anal. calcd for $\mathrm{C}_{22} \mathrm{H}_{27} \mathrm{BrN}_{2}$ : C 66.16, H 6.81, N 7.01; found C 66.02, H 6.95, N 6.83.

3-(4'-氟苯基)-5,5,9,9-四甲基-2,4,5,6,7,8,9,9a-八 氢-5a,8-亚甲基桥苯并吲唑 $(3 \mathrm{c})$ ：得率 $71.8 \%$. 白色棉状 固体, m.p. 260.7 261.3 ${ }^{\circ} \mathrm{C} ;{ }^{1} \mathrm{H}$ NMR (DMSO- $d_{6}, 500$ $\mathrm{MHz}) \delta$ : $0.66(\mathrm{~s}, 3 \mathrm{H}), 0.75$ (s, 3H), 1.04 (s, 3H), 1.15 (s, $1 \mathrm{H}), 1.20(\mathrm{~d}, J=9.4 \mathrm{~Hz}, 1 \mathrm{H}), 1.25(\mathrm{~s}, 3 \mathrm{H}), 1.44 \sim 1.48(\mathrm{~m}$, $1 \mathrm{H}), 1.57(\mathrm{~d}, J=9.3 \mathrm{~Hz}, 1 \mathrm{H}), 1.67 \sim 1.69(\mathrm{~m}, 2 \mathrm{H}), 1.80 \sim$ $1.82(\mathrm{~m}, 1 \mathrm{H}), 2.23(\mathrm{~s}, 1 \mathrm{H}), 2.30(\mathrm{~d}, J=15.1 \mathrm{~Hz}, 1 \mathrm{H}), 2.62$ $(\mathrm{d}, J=15.0 \mathrm{~Hz}, 1 \mathrm{H}), 7.06\left(\mathrm{t}, J_{\mathrm{H}-\mathrm{F}}=9.0 \mathrm{~Hz}, 2 \mathrm{H}\right), 7.66(\mathrm{t}$, $J_{\mathrm{H}-\mathrm{F}}=7.2 \mathrm{~Hz}, 2 \mathrm{H}$ ), 12.40 (brs, $1 \mathrm{H}, \mathrm{NH}$ ); ${ }^{13} \mathrm{C} \mathrm{NMR}$ $\left(\mathrm{CDCl}_{3}, 125 \mathrm{MHz}\right) \delta: 23.45,25.31,26.00,27.13,28.11$, $29.56,34.21,35.96,36.71,42.10,48.33,50.69$, 56.47, $111.73,115.28\left(\mathrm{~d}, J_{\mathrm{H}-\mathrm{F}}=21.6 \mathrm{~Hz}, 1 \mathrm{C}\right), 128.57\left(\mathrm{~d}, J_{\mathrm{H}-\mathrm{F}}=\right.$ $8.7 \mathrm{~Hz}, 1 \mathrm{C}), 162.16\left(\mathrm{~d}, J_{\mathrm{C}-\mathrm{F}}=244.8 \mathrm{~Hz}, 1 \mathrm{C}\right)$; IR (KBr) v: 3223, 2968, 2874, 1505, 1461, 1367, 1232, $835 \mathrm{~cm}^{-1}$; MS (70 eV) m/z (\%): $338\left(\mathrm{M}^{+}, 100\right), 323$ (17), 255 (99). Anal. calcd for $\mathrm{C}_{22} \mathrm{H}_{27} \mathrm{FN}_{2}$ : C 78.07, $\mathrm{H} \mathrm{8.04,} \mathrm{N} 8.28$; found $\mathrm{C}$ 
78.08, H 8.12, N 8.27.

5,5,9,9-四甲基-3-对甲苯基- $2,4,5,6,7,8,9,9 \mathrm{a}$-八 氢-5a,8-亚甲基桥苯并吲唑 $(\mathbf{3 d})$ : 得率 $69.5 \%$. 淡白色棉 状固体, m.p. $220.4 \sim 221.0{ }^{\circ} \mathrm{C} ;{ }^{1} \mathrm{H}$ NMR (DMSO- $d_{6}, 500$ MHz) $\delta$ : 0.65 (s, 3H), 0.75 (s, 3H), 1.04 (s, 3H), 1.15 (s, $1 \mathrm{H}), 1.20(\mathrm{~d}, J=9.8 \mathrm{~Hz}, 1 \mathrm{H}), 1.25(\mathrm{~s}, 3 \mathrm{H}), 1.44 \sim 1.48(\mathrm{~m}$, $1 \mathrm{H}), 1.58(\mathrm{~d}, J=9.5 \mathrm{~Hz}, 1 \mathrm{H}), 1.67 \sim 1.70(\mathrm{~m}, 2 \mathrm{H}), 1.80 \sim$ $1.84(\mathrm{~m}, 1 \mathrm{H}), 2.22(\mathrm{~s}, 1 \mathrm{H}), 2.28 \sim 2.32(\mathrm{~m}, 4 \mathrm{H}), 2.62(\mathrm{~d}$, $J=15.2 \mathrm{~Hz}, 1 \mathrm{H}), 7.21(\mathrm{~d}, J=7.0 \mathrm{~Hz}, 2 \mathrm{H}), 7.52(\mathrm{~s}, 2 \mathrm{H})$, 12.32 (brs, $1 \mathrm{H}, \mathrm{NH}) ;{ }^{13} \mathrm{C} \mathrm{NMR}\left(\mathrm{CDCl}_{3}, 75 \mathrm{MHz}\right) \delta: 21.23$, $23.48,25.36,26.04,27.21,28.33,29.62,34.13,35.94$, $36.74,42.14,48.33,50.90,56.46,111.75,126.71,129.17$, 136.90; IR (KBr) v: 3241, 2969, 2877, 1504, 1466, 1383, 1360, 1319, $819 \mathrm{~cm}^{-1}$; MS (70 eV) m/z (\%): $334\left(\mathrm{M}^{+}\right.$, 100), 319 (17), 251 (57). Anal. calcd for $\mathrm{C}_{23} \mathrm{H}_{30} \mathrm{~N}_{2}$ : C 82.59, H 9.04, N 8.37; found C 82.71, H 9.06, N 8.21.

5,5,9,9-四甲基-3-(4'-甲氧基苯基)-2,4,5,6,7,8,9,9a-八 氢-5a,8-亚甲基桥苯并吲唑 $(3 \mathrm{e})$ : 得率 74.6\%. 淡白色块 状晶体, m.p. $227.6 \sim 228.3{ }^{\circ} \mathrm{C} ;{ }^{1} \mathrm{H}$ NMR (DMSO- $d_{6}, 500$ MHz) $\delta: 0.65$ (s, 3H), 0.75 (s, 3H), 1.04 (s, 3H), 1.15 (s, $1 \mathrm{H}), 1.20(\mathrm{~d}, J=9.7 \mathrm{~Hz}, 1 \mathrm{H}), 1.24(\mathrm{~s}, 3 \mathrm{H}), 1.43 \sim 1.48(\mathrm{~m}$, $1 \mathrm{H}), 1.58(\mathrm{~d}, J=9.6 \mathrm{~Hz}, 1 \mathrm{H}), 1.67 \sim 1.69(\mathrm{~m}, 2 \mathrm{H}), 1.80 \sim$ $1.84(\mathrm{~m}, 1 \mathrm{H}), 2.22(\mathrm{~s}, 1 \mathrm{H}), 2.28(\mathrm{~d}, J=15.1 \mathrm{~Hz}, 1 \mathrm{H}), 2.60$ (d, $J=15.0 \mathrm{~Hz}, 1 \mathrm{H}), 3.78$ (s, 3H, Ar-OCH $), 6.98$ (d, $J=$ $8.1 \mathrm{~Hz}, 2 \mathrm{H}$ ), 7.55 (d, $J=6.9 \mathrm{~Hz}, 2 \mathrm{H}$ ), 12.26 (brs, $1 \mathrm{H}, \mathrm{NH}$ ); ${ }^{13} \mathrm{C}$ NMR $\left(\mathrm{CDCl}_{3}, 75 \mathrm{MHz}\right) \delta: 23.50,25.36,26.03,27.22$, $28.37,28.44,29.63,34.15,35.94,36.75,42.14,48.33$, $50.91,55.28,56.47,111.43,113.94,128.02,158.92$; IR (KBr) v: 3114, 3061, 3035, 2964, 2922, 2898, 2841, 1616, $1534,1462,1248,1034,830 \mathrm{~cm}^{-1}$; MS (70 eV) $\mathrm{m} / z(\%)$ : $350\left(\mathrm{M}^{+}, 100\right), 335$ (13), 268 (26). Anal. calcd for $\mathrm{C}_{23} \mathrm{H}_{30} \mathrm{~N}_{2} \mathrm{O}$ : C 78.82, $\mathrm{H} \mathrm{8.63,} \mathrm{N} \mathrm{7.99;} \mathrm{found} \mathrm{C} 78.75, \mathrm{H}$ 8.78, N 7.97.

5,5,9,9-四甲基-3-苯基-2,4,5,6,7,8,9,9a-八氢- $5 \mathrm{a}, 8$-亚 甲基桥苯并吲唑(3f): 得率 70.4\%. 白色固体, m.p. $229.5 \sim 230.1{ }^{\circ} \mathrm{C} ;{ }^{1} \mathrm{H}$ NMR (DMSO- $\left.d_{6}, 500 \mathrm{MHz}\right) \delta: 0.66$ (s, 3H), $0.76(\mathrm{~s}, 3 \mathrm{H}), 1.05(\mathrm{~s}, 3 \mathrm{H}), 1.13 \sim 1.18(\mathrm{~m}, 1 \mathrm{H})$, $1.21(\mathrm{~d}, J=9.7 \mathrm{~Hz}, 1 \mathrm{H}), 1.25(\mathrm{~s}, 3 \mathrm{H}), 1.44 \sim 1.49(\mathrm{~m}, 1 \mathrm{H})$, $1.59(\mathrm{~d}, J=9.7 \mathrm{~Hz}, 1 \mathrm{H}), 1.67 \sim 1.70(\mathrm{~m}, 2 \mathrm{H}), 1.80 \sim 1.84$ (m, 1H), 2.24 (s, 1H), 2.33 (d, $J=15.1 \mathrm{~Hz}, 1 \mathrm{H}), 2.64$ (d, $J=15.1 \mathrm{~Hz}, 1 \mathrm{H}), 7.26 \sim 7.29(\mathrm{~m}, 2 \mathrm{H}), 7.39 \sim 7.42(\mathrm{~m}, 2 \mathrm{H})$, 12.39 (brs, $1 \mathrm{H}, \mathrm{NH}) ;{ }^{13} \mathrm{C} \mathrm{NMR}\left(\mathrm{CDCl}_{3}, 75 \mathrm{MHz}\right) \delta: 18.38$, $23.47,25.34,26.02,27.15,28.23,29.59,34.14,35.94$, $36.72,42.13,48.32,50.81,56.46,58.22,111.95,126.82$, 127.17, 128.44, 133.21; IR (KBr) v: 3217, 3062, 2968,
2921, 2876, 2836, 1498, 1463, 768, $694 \mathrm{~cm}^{-1}$; MS (70 eV) $\mathrm{m} / z$ (\%): $320\left(\mathrm{M}^{+}, 100\right), 305$ (18), 277 (31). Anal. calcd for $\mathrm{C}_{22} \mathrm{H}_{28} \mathrm{~N}_{2}$ : C 82.45, H 8.81, N 8.74; found C 82.32, H 8.83, N 8.85 .

5,5,9,9-四甲基-3-(4'-硝基苯基)-2,4,5,6,7,8,9,9a-八 氢-5a,8-亚甲基桥苯并吲唑 $(\mathbf{3 g})$ : 得率 $72.9 \%$. 黄色块状 固体, m.p. $229.6 \sim 230.3{ }^{\circ} \mathrm{C}$; ${ }^{1} \mathrm{H}$ NMR (DMSO- $d_{6}, 500$ MHz) $\delta: 0.67$ (s, 3H), 0.75 (s, 3H), 1.07 (s, 3H), 1.14 $1.19(\mathrm{~m}, 1 \mathrm{H}), 1.22(\mathrm{~d}, J=9.8 \mathrm{~Hz}, 1 \mathrm{H}), 1.27(\mathrm{~s}, 3 \mathrm{H}), 1.44 \sim$ $1.50(\mathrm{~m}, 1 \mathrm{H}), 1.58(\mathrm{~d}, J=9.6 \mathrm{~Hz}, 1 \mathrm{H}), 1.68 \sim 1.73(\mathrm{~m}$, $2 \mathrm{H}), 1.81 \sim 1.85(\mathrm{~m}, 1 \mathrm{H}), 2.26(\mathrm{~s}, 1 \mathrm{H}), 2.40(\mathrm{~d}, J=15.2$ $\mathrm{Hz}, 1 \mathrm{H}), 2.70(\mathrm{~d}, J=15.2 \mathrm{~Hz}, 1 \mathrm{H}), 7.93(\mathrm{~d}, J=8.2 \mathrm{~Hz}$, $2 \mathrm{H}), 8.27$ (d, $J=8.7 \mathrm{~Hz}, 2 \mathrm{H}), 12.77$ (brs, $1 \mathrm{H}, \mathrm{NH}) ;{ }^{13} \mathrm{C}$ NMR $\left(\mathrm{CDCl}_{3}, 75 \mathrm{MHz}\right) \delta: 23.48,25.27,25.96,27.20$, 28.33, 29.47, 34.28, 36.06, 36.72, 42.10, 48.31, 50.45, $56.46,113.21,123.84,127.23,140.34,146.64$; IR (KBr) v: 3401, 2963, 2924, 2896, 2873, 1598, 1465, 1518, 1340, $855 \mathrm{~cm}^{-1}$; MS (70 eV) $\mathrm{m} / z(\%): 365\left(\mathrm{M}^{+}, 59\right), 350(10)$, 282 (100). Anal. calcd for $\mathrm{C}_{22} \mathrm{H}_{27} \mathrm{~N}_{3} \mathrm{O}_{2}: \mathrm{C} 72.30, \mathrm{H} 7.45, \mathrm{~N}$ 11.50; found C 72.21, H 7.43, N 11.67.

5,5,9,9-四甲基-3-(3'-硝基苯基)-2,4,5,6,7,8,9,9a-八 氢-5a,8-亚甲基桥苯并吲唑(3h): 得率 70.7\%. 黄色棉状 固体, m.p. $250.2 \sim 250.6{ }^{\circ} \mathrm{C} ;{ }^{1} \mathrm{H}$ NMR (DMSO- $d_{6}, 500$ MHz) $\delta: 0.67$ (s, 3H), $0.76(\mathrm{~s}, 3 \mathrm{H}), 1.07$ (s, 3H), 1.14 $1.19(\mathrm{~m}, 1 \mathrm{H}), 1.22(\mathrm{~d}, J=9.7 \mathrm{~Hz}, 1 \mathrm{H}), 1.27(\mathrm{~s}, 3 \mathrm{H}), 1.44 \sim$ $1.50(\mathrm{~m}, 1 \mathrm{H}), 1.59(\mathrm{~d}, J=9.6 \mathrm{~Hz}, 1 \mathrm{H}), 1.68 \sim 1.73(\mathrm{~m}$, $2 \mathrm{H}), 1.81 \sim 1.85(\mathrm{~m}, 1 \mathrm{H}), 2.26(\mathrm{~s}, 1 \mathrm{H}), 2.38(\mathrm{~d}, J=15.1$ $\mathrm{Hz}, 1 \mathrm{H}), 2.70(\mathrm{~d}, J=15.1 \mathrm{~Hz}, 1 \mathrm{H}), 7.70 \sim 7.73(\mathrm{~m}, 1 \mathrm{H})$, $7.72(\mathrm{~d}, J=8.0 \mathrm{~Hz}, 1 \mathrm{H}), 8.13$ (d, $J=8.0 \mathrm{~Hz}, 1 \mathrm{H}), 8.46$ (s, $1 \mathrm{H}), 12.67$ (brs, $1 \mathrm{H}, \mathrm{NH}) ;{ }^{13} \mathrm{C} \mathrm{NMR}\left(\mathrm{CDCl}_{3}, 75 \mathrm{MHz}\right) \delta$ : $23.27,25.27,25.96,27.18,28.27,29.47,34.28,35.76$, $36.73,42.09,48.30,50.46,56.49,112.54,121.63,121.78$, 129.30, 132.72, 135.70, 148.48; IR (KBr) v: 3180, 3119, 3054, 2965, 2880, 1588, 1466, 1534, 1348, 867, 803, 723 $\mathrm{cm}^{-1}$; MS (70 eV) m/z (\%): $365\left(\mathrm{M}^{+}, 57\right), 350(9), 282$ (100). Anal. calcd for $\mathrm{C}_{22} \mathrm{H}_{27} \mathrm{~N}_{3} \mathrm{O}_{2}$ : C 72.30, H 7.45, N 11.50; found C 72.40, H 7.48, N 11.41.

5,5,9,9-四甲基-3-(4'-二甲氨基苯基)-2,4,5，6,7,8,9， 9a-八氢-5a,8-亚甲基桥苯并吲唑(3i): 得率 73.1\%. 褐色 块状固体, m.p. $198.3 \sim 199.1{ }^{\circ} \mathrm{C} ;{ }^{1} \mathrm{H}$ NMR (DMSO- $d_{6}$, $500 \mathrm{MHz}) \delta: 0.65(\mathrm{~s}, 3 \mathrm{H}), 0.76(\mathrm{~s}, 3 \mathrm{H}), 1.04(\mathrm{~s}, 3 \mathrm{H})$, $1.11 \sim 1.17(\mathrm{~m}, 1 \mathrm{H}), 1.19(\mathrm{~d}, J=9.7 \mathrm{~Hz}, 1 \mathrm{H}), 1.24(\mathrm{~s}, 3 \mathrm{H})$, $1.42 \sim 1.49(\mathrm{~m}, 1 \mathrm{H}), 1.58(\mathrm{~d}, J=9.5 \mathrm{~Hz}, 1 \mathrm{H}), 1.66 \sim 1.71$ $(\mathrm{m}, 2 \mathrm{H}), 1.80 \sim 1.84(\mathrm{~m}, 1 \mathrm{H}), 2.21(\mathrm{~s}, 1 \mathrm{H}), 2.28(\mathrm{~d}, J=$ $15.1 \mathrm{~Hz}, 1 \mathrm{H}), 2.59(\mathrm{~d}, J=15.0 \mathrm{~Hz}, 1 \mathrm{H}), 2.91(\mathrm{~s}, 6 \mathrm{H}$, 
$\left.\mathrm{N}\left(\mathrm{CH}_{3}\right)_{2}\right), 6.76(\mathrm{~d}, J=8.7 \mathrm{~Hz}, 2 \mathrm{H}), 7.45(\mathrm{~d}, J=8.3 \mathrm{~Hz}$, $2 \mathrm{H}), 12.22$ (brs, $1 \mathrm{H}, \mathrm{NH}) ;{ }^{13} \mathrm{C}$ NMR $\left(\mathrm{CDCl}_{3}, 75 \mathrm{MHz}\right) \delta$ : $23.51,25.37,26.06,27.15,28.30,28.37,29.70,34.09$, $35.95,36.74,40.50,42.17,48.33,51.06,56.43,110.98$, 112.45, 127.61, 149.79; IR (KBr) v: 3233, 2962, 2872, $1616,1538,1462,1439,1357,815 \mathrm{~cm}^{-1}$; MS (70 eV) $\mathrm{m} / \mathrm{z}$ (\%): $363\left(\mathrm{M}^{+}, 100\right), 348(6), 281$ (15). Anal. calcd for $\mathrm{C}_{24} \mathrm{H}_{33} \mathrm{~N}_{3}$ : C 79.29, H 9.15, N 11.56; found C 79.13, H 9.21, N 11.66 .

5,5,9,9-四甲基-3-(2',4'-二氯苯基)-2,4,5,6,7,8,9,9a-八 氢-5a,8-亚甲基桥苯并吲唑 $(\mathbf{3 j})$ : 得率 76.5\%. 白色块状 晶体, m.p. $255.4 \sim 255.8{ }^{\circ} \mathrm{C} ;{ }^{1} \mathrm{H}$ NMR (DMSO- $d_{6}, 500$ MHz) $\delta: 0.64$ (s, 3H), 0.77 (s, 3H), 0.98 (s, 3H), 1.12 $1.15(\mathrm{~m}, 1 \mathrm{H}), 1.19(\mathrm{~d}, J=9.9 \mathrm{~Hz}, 1 \mathrm{H}), 1.26(\mathrm{~s}, 3 \mathrm{H}), 1.43 \sim$ $1.48(\mathrm{~m}, 1 \mathrm{H}), 1.56(\mathrm{~d}, J=9.5 \mathrm{~Hz}, 1 \mathrm{H}), 1.65 \sim 1.70(\mathrm{~m}$, $2 \mathrm{H}), 1.80 \sim 1.84(\mathrm{~m}, 1 \mathrm{H}), 1.93(\mathrm{~d}, J=15.1 \mathrm{~Hz}, 1 \mathrm{H}), 2.25$ (s, 1H), $2.42(\mathrm{~d}, J=15.1 \mathrm{~Hz}, 1 \mathrm{H}), 7.42 \sim 7.46(\mathrm{~m}, 2 \mathrm{H})$, 7.66 (m, 1H), 12.52 (brs, $1 \mathrm{H}, \mathrm{NH}) ;{ }^{13} \mathrm{C}$ NMR $\left(\mathrm{CDCl}_{3}, 75\right.$ MHz) $\delta: 23.27,25.31,25.89,27.15,28.23,29.56,34.12$, $35.09,36.72,42.06,48.19,50.85,56.64,114.20,126.81$, 129.58, 132.44, 134.18; IR (KBr) v: 3175, 3117, 3053, 2966, 2927, 2875, 1591, 1546, 1464, 1446, 1380, 1364, 1319, 867, $809 \mathrm{~cm}^{-1}$; MS (70 eV) $\mathrm{m} / z(\%): 389\left(\mathrm{M}^{+}, 29\right)$, 374 (4), 305 (100), Anal. calcd for $\mathrm{C}_{22} \mathrm{H}_{26} \mathrm{Cl}_{2} \mathrm{~N}_{2}$ : C 67.87, H 6.73, N 7.19; found C 68.01, H 6.71, N 7.29.

\subsection{3 晶体结构测定}

为了对该系列化合物分子结构作进一步的验证, 选 取化合物 3i 进行了 X 射线单晶衍射分析.

将经过乙醇重结晶后的目标化合物 $\mathbf{3 i}$ 溶于乙醇中, 室温静置使其慢慢挥发, 数天后形成淡黄色晶体, 选取 尺寸为 $0.35 \mathrm{~mm} \times 0.20 \mathrm{~mm} \times 0.20 \mathrm{~mm}$ 的晶体进行 $X$ 射 线衍射分析. 结果表明, 化合物晶体为正交晶系, 空间 群 $\mathrm{P} 21212$, 晶胞参数为 $a=19.0581(13) \AA, b=$ 10.8438(8) $\AA, c=10.844 \AA, \alpha=90^{\circ}, \beta=90^{\circ}, \gamma=90^{\circ}, Z=$ 2, $V=2241.0(2) \AA^{3}, D_{\mathrm{c}}=1.140 \mathrm{Mg} / \mathrm{m}^{3}, \mu=0.068 \mathrm{~mm}^{-1}$, $F(000)=836$. 数据存于英国剑桥数据中心, $\mathrm{CCDC}$ 号为 1056456 .

\subsection{4 化合物的生物活性测试}

人脐静脉内皮细胞(HUVECs)培养方法: 用含 $10 \%$ 小牛血清的 DMEM 培养基培养人脐静脉内皮细胞, 细 胞放在 $\mathrm{CO}_{2}$ 餒育箱中培养(其条件为 $37{ }^{\circ} \mathrm{C}, 5 \% \mathrm{CO}_{2}, 95 \%$ 空气, 保持一定湿度), 将细胞置于显微镜下以便于观 察细胞生长情况, 依据细胞的生长情况, 通过换液; 培 养 24 48 h 后, 细胞即可生长成单层, 然后用 $0.25 \%$ 胰 蛋白酶消化, $1: 3$ 传代. 实验过程中采用生长成单层的
人脐静脉内皮细胞.

肝癌细胞(HepG2)培养方法: 用含 $10 \%$ 小牛血清的 DMEM 培养基培养肝癌细胞( $\mathrm{HepG} 2$ ), 细胞放在 $\mathrm{CO}_{2}$ 捊 育箱中培养 $\left(37{ }^{\circ} \mathrm{C}, 5 \% \mathrm{CO}_{2}, 95 \%\right.$ 空气、保持一定湿度), 将细胞置显微镜下观察细胞的生长情况; 培养 $24 \sim 48 \mathrm{~h}$ 后, 细胞即可生长成单层, 然后用 $0.25 \%$ 胰蛋白酶消化, 按 $1: 2$ 传代. 实验过程中采用生长成单层的肝癌细胞 (HepG2).

药品溶液的配制: 将目标化合物用 DMSO 配制成 一定浓度的母液, 再用磷酸盐缓冲液(PBS)将衍生物母 液稀释成不同作用浓度梯度的稀释液, 浓度分别为 1 , $10,100,500,1000 \mu \mathrm{mol} / \mathrm{L}$. 移去旧培养基, 加入不同浓 度的含药培养基, 每孔 $200 \mu \mathrm{L}$. 另设空白对照组和阳性 对照阿司匹林和依托泊苷对照组. 细胞在药物培养基中 培养 $24 \mathrm{~h}$ 后，吸弃含药培养基.

MTT 比色法抗肿瘤活性测试: 将 $10 \mu \mathrm{L}$ 浓度为 5 $\mathrm{mg} / \mathrm{mL}$ 噻唑蓝(MTT)溶液加入到酶标板孔内, 在 $37{ }^{\circ} \mathrm{C}$ 培养箱中孵育 $4 \mathrm{~h}$, 离心后除去上清液, 分别加入 150 $\mu \mathrm{L}$ DMSO 溶液，振荡 $10 \mathrm{~min}$, 待固体结晶溶解完全，将 酶标仪的波长 $\lambda$ 设定为 $490 \mathrm{~nm}$, 然后测定溶液的吸光度 值. 利用各孔的光吸收值 (OD 值), 计算细胞的增殖抑制 率:

$$
I(\%)=\left(1-\mathrm{OD}_{1} / \mathrm{OD}\right) \times 100 \%
$$

其中, $I$ 为细胞的抑制率; $\mathrm{OD}_{1}$ 为加药组细胞吸光度值(平 行试验三次取平均值), $\mathrm{OD}$ 为空白对照组细胞吸光度值 (平行试验三次取平均值), 最后将所得的增殖抑制率换 算成 $\mathrm{IC}_{50}$ 值(诱导细胞调亡 $50 \%$ 的浓度).

辅助材料(Supporting Information) 化合物 $\mathbf{2 a} \sim \mathbf{2 j}$ 与 $3 \mathbf{a} \sim 3 \mathbf{j}$ 化合物的氢谱、碳谱、气质谱图以及元素分析具 体数据. 这些材料可以免费从本刊网站 (http://siocjournal.cn/)上下载.

\section{References}

[1] Krishnaiah, A.; Narsaiah, B. J. Fluorine Chem. 2002, 115, 9.

[2] Du, H. T.; Du, H. J.; Ouyang, G. P. Chin. J. Org. Chem. 2008, 28, 356 (in Chinese). (杜海堂, 杜海军, 欧阳贵平, 有机化学, 2008, 28, 356.)

[3] Tong, J. Y.; Shi, Y. X.; Liu, X. H.; Sun, N. B.; Li, B. J. Chin. J. Org. Chem. 2012, 32, 2373 (in Chinese). (童建颖, 石延霞, 刘幸海, 孙娜波, 李宝聚, 有机化学, 2012, 32, 2373.)

[4] Nasr, T.; Bondock, S.; Eid, S. Eur. J. Med. Chem. 2014, 84, 492.

[5] Fadda, A. A.; El-Mekawy, R. E.; El-Shafei, A. I. J. Porphyrins Phthalocyanines 2015, 19, 754.

[6] Palanisamy, P.; Kumaresan, S. RSC Adv. 2013, 3, 4705.

[7] Gökhan-Kelekçi, N.; Yabanoğlu, S.; Küpeli, E. Bioorg. Med. Chem. 2007, 15, 5775 . 
[8] Lv, P. C.; Li, H. Q.; Sun, J.; Zhou, Y.; Zhu, H. L. Bioorg. Med. Chem. 2010, 18, 4606

[9] Goda, F. E.; Maarouf, A. R.; El-Bendary, E. R. Saudi Pharm. J. 2003, 11,111

[10] Rüger, A. J.; Nieger, M.; Bräse, S. Tetrahedron 2012, 68, 8824.

[11] Dai, H.; Chen, J.; Li, H.; Dai, B. J.; He, H. B.; Fang, Y.; Shi, Y. J. Molecules 2016, 21, 276.

[12] Foster, R. S.; Jakobi, H.; Harrity, J. P. A. Org. Lett. 2012, 14, 4859.

[13] Kawamori, T.; Rao, C. V.; Seibert, K.; Reddy, B. S. Cancer Res. 1998, 58, 409.

[14] Hou, Q. Q.; Li, Z. N.; Liu, C. L. Pesticides 2002, 41, 41 (in Chinese)

(侯春青, 李志念, 刘长令, 农药, 2002, 41, 41.)

[15] Kubota, M.; Yamamoto, K. JP 10130267, 1998 [Chem. Abstr. 1998, 129, 41126.]

[16] Gordien, A. Y.; Gray, A. I.; Franzblau, S. G.; Seidel, V. J. Ethnopharmacol. 2009, 126, 500 .

[17] Bichlmaier, I.; Kurkela, M.; Siiskonen, A.; Finel, M.; Kauhaluoma, J. Y. Bioorg. Med. Chem. 2007, 35, 386
[18] Zhang, A. J.; Klun, J. A.; Wang, S. F. J. Med. Entomol. 2009, 46, 100 .

[19] Zhang, A.; Carroll, J.; Wang, S.; Klun, J. A. WO 200901209, 2009 [Chem. Abstr. 2009, 150, 144686.]

[20] El-Sayed, M. A.; Abdel-Aziz, N. I.; Abdel-Aziz, A. A.; El-Azab, A. S.; ElTahir, K. E. H. Bioorg. Med. Chem. 2012, 20, 3306.

[21] Thurmond, R. L.; Beavers, M. P.; Cai, H.; Meduna, S. P.; Gustin, D. J.; Sun, S.; Almond. H. J.; Karlsson, L.; Edwards, J. P. J. Med. Chem. 2004, 47,4799.

[22] Dinges, J.; Albert, D. H.; Arnold, L. D.; Ashworth, K. L.; Akritopoulou-Zanze, I.; Bousquet, P. F.; Bouska, J. J.; Cunha, G. A.; Davidsen, S. K.; Diaz, G. J.; Djuric, S. W.; Gasiecki, A. F.; Gintant, G. A.; Gracias, V. J.; Harris, C. M.; Houseman, K. A.; Huthins, C. W.; Johnson, E. F.; Li, H.; Marcotte, P. A.; Martin, R. L.; Michaelids, M. R.; Nyein, M.; Sowin, T. J.; Su, Z.; Tapang, P. H.; Xia, Z.; Zhang, H. Q. J. Med. Chem. 2007, 50, 2011.

[23] Klein, O.; Aguilar-Parrilla, F.; Lopez, J. M.; Jagerovic, N.; Elguero, J.; Limbach, H. H. J. Am. Chem. Soc. 2004, 126, 11718. 